\title{
Characterization of surface topography of a newly developed metrological gloss scale.
}

\author{
O Flys $^{1,2}$, S Källberg ${ }^{2}$, G Ged ${ }^{3}$, Z Silvestri ${ }^{3}$, B-G Rosén ${ }^{1}$ \\ ${ }^{1}$ School of Business and Engineering, Halmstad University, PO Box 823, SE-301 18 \\ Halmstad, Sweden; \\ ${ }^{2}$ SP Technical Research Institute of Sweden, Box 857, SE-501 15 Borås; \\ ${ }^{3}$ LCM LNE-Cnam, 29 avenue Roger Hennequin, 78190, Trappes, France;
}

olena.flys@hh.se

\begin{abstract}
In the European Joint Research Project "Multidimensional reflectometry for industry", a new gloss scale has been developed with the aim to represent different levels of gloss, hue, roughness and refractive indices. In this paper, the surfaces of six selected samples have been thoroughly investigated using various measuring techniques in order to verify the outcome of the novel manufacturing processes in terms of distinct levels as well as types of surface roughness. The aim of the evaluation was to capture surface structures in different wavelength intervals utilizing a confocal microscope, a coherence scanning interferometer and an atomic force microscope. Also, PSD functions calculated from the measurements have been used to determine suitability of techniques for different roughness scales. Measurements show the expected surface characteristics as well as different rms roughness values intimately connected to the perceived glossiness.
\end{abstract}

\section{Introduction}

Gloss is a visual attribute that is recognized as the second most relevant beside colour [1,2], and is often associated with the notion of quality and thus, for many products, acceptability. Surface roughness and glossiness are strongly correlated [3] and it is therefore of interest to relate the visual gloss of material with the surface structure. Surface features on nano- and micro scale are known to influence gloss, haze and DOI (distinctness of image) [4] and therefore different measurement techniques may need to be used in order to capture all important surface properties.

Nowadays the most common instrument used for measurement of gloss is the glossmeter, an instrument based on the ISO 2813 standard. Such a device measures gloss indices which are not linearly linked to the perceived visual gloss [5]. Furthermore, glossmeters are not able to extensively describe physical phenomena that may be used as stepping stone by the visual system to establish its perception of gloss, such as the topology of the specular peak or the coloured appearance of the sample under test.

Comprising review of recent research in the field of gloss perception has been made by Chadwick and Kentridge [6]. It been pointed out that perceptual gloss is reliant in multiple dimensions and factors such as illumination, specular reflectance, surface textures and shape, surface colour, viewing distance, binocular disparity, physical interaction should be taken in to account.

The aim of this study was to find the influence of surface structure on micro and nanoscale level 
on gloss. Bennett and Mattsson [7] described influence of surface structure on specular reflection of scattered light and showed that light scattering from the surface micro roughness gives information of surface indirectly and theory is needed to relate surface roughness to scattered light. Qi et al.[8] investigated how mesoscale and microscale roughness affect perceived roughness, where difference between mesoscale and microscale can be compared as pebbledash and sandpaper. As well as study performed by Ho, Landy and Maloney [9] their study shows non-linear effect of surface structure on perceived gloss.

In order to separate different components in the measured data different filtration techniques are widely used. The Gaussian filter as a typical mean-line based filter is often used but not suitable for all types of surfaces especially for surfaces with very close wavelength for roughness and waviness.

Motif method is an alternative method that can separate surface components from unfiltered data [10]. This method was developed and widely used by French automotive industry and later became the basis for the parameter adopted by ISO 12085 [11]. Areal motif methods provide useful tools for the evaluation of surface geometry. The wolf pruning techniques is used as criterion and in combination with areal motif creates stability of motif method [12]. In the present study motif analysis is proposed to illustrate the surface structure of samples.

In the joint research project Multidimensional Reflectometry for Industry [13], a part of the ongoing European Metrology Research Programme (EMRP), a new method for manufacturing surfaces with different glossiness, roughness, colour and refractive indices with the aim to develop a new gloss scale, have been developed. Since gloss is strongly correlated with the roughness of a surface, the measurement and the characterization of surfaces with different gloss levels were examined in this paper. As both nano- and microscopic surface features are known to influence the perceived gloss, different measurements techniques were used in order to capture most of all possibly important surface properties. A study by A. Duparré et al. demonstrates the usefulness of Power Spectral Density functions (PSDs) which enables a combination of the results obtained using different roughness measuring techniques [14]. The PSD, which is merely the square of the surface profile's Fourier spectrum, contains essential (except phase) information about both the vertical and the lateral structural properties. In this paper PSDs have been used for characterisation of the surfaces in different spatial frequency ranges.

\section{Materials and methods}

\subsection{Samples}

Six different samples were used for investigation in this study. The samples were selected in order to be able to systematically compare the many different variations of the final gloss standards. Table 1 shows an overview of the selected and studied samples.

Table 1. Classification of the samples based on gloss, roughness and refractive index.

\begin{tabular}{lccc}
\hline $\begin{array}{l}\text { Samples } \\
\text { abbreviation }\end{array}$ & $\mathrm{R}_{3} \mathrm{n}_{2} \mathrm{G}_{4} / \mathrm{R}_{3} \mathrm{n}_{1} \mathrm{G}_{4}$ & $\mathrm{R}_{2} \mathrm{n}_{2} \mathrm{G}_{3} / \mathrm{R}_{1} \mathrm{n}_{2} \mathrm{G}_{3}$ & $\mathrm{R}_{1} \mathrm{n}_{2} \mathrm{G}_{1} / \mathrm{R}_{1} \mathrm{n}_{1} \mathrm{G}_{1}$ \\
\hline Roughness & - Same type $\left(\mathrm{R}_{3}\right)$ & - Different types $\left(\mathrm{R}_{1}, \mathrm{R}_{2}\right)$ & - Same type $\left(\mathrm{R}_{1}\right)$ \\
$\begin{array}{l}\text { Gloss } \\
\text { Index }\end{array}$ & - Same level $\left(\mathrm{G}_{4}\right)$ & - Same level $\left(\mathrm{G}_{3}\right)$ & - Same level $\left(\mathrm{G}_{1}\right)$ \\
- Different index $\left(\mathrm{n}_{1}, \mathrm{n}_{2}\right)$ & - Same index $\left(\mathrm{n}_{2}\right)$ & - Different index $\left(\mathrm{n}_{1}, \mathrm{n}_{2}\right)$
\end{tabular}

The evaluated samples have been manufactured using sol-gel chemistry for accurate replication of surface structures [15]. The samples' gloss abbreviation G1, G3, G4 used in the paper was decided by the manufacturing process used in the EU project introduced in the abstract. The samples manufacturing process have been tuned to generate different reflectance of illuminated light correlating to the perceived gloss as discussed and referenced to in the article [7-9].

High gloss samples $\left(G_{4}\right.$ with surface roughness type $\left.R_{3}\right)$ include two samples, one for each refractive index $\left(R_{3} n_{1} G_{4}\right.$ and $\left.R_{3} n_{2} G_{4}\right)$. These samples were produced by sol-gel deposited on silicon 
wafer using a spin coater in a clean environment. Samples of the $\mathrm{R}_{1}$-type were produced in two steps, first by making replications in elastomeric polymer mould and then by imprinting the replicas in tuned silica sol-gel with different refractive index $\left(R_{1} n_{1} G_{1}, R_{1} n_{2} G_{1}\right.$ and $\left.R_{1} n_{2} G_{3}\right)$. Similar to the previous group $R_{1}$, samples of type $R_{2}$ were manufactured in two steps pre-replicas were imprinted in thermoplastic polymers. They underwent controlled thermal relaxation processes in order to achieve different gloss levels $\left(\mathrm{R}_{2} \mathrm{n}_{2} \mathrm{G}_{3}\right)$ and were finally replicated into tuned sol gel. Then the samples underwent controlled thermal relaxation processes in order to achieve different gloss levels $\left(R_{2} n_{2} G_{3}\right)$. Note that the samples in this study were manufactured on silicon substrates and in that aspect differ from the samples used for the actual gloss scale which are made of glass (whose back is painted white, grey or black). The reason for this was to avoid possible measuring artefact due to glass reflections when using optical measurement techniques.

\subsection{Instruments}

The instruments and measurement techniques were chosen in order to be able to describe the surface structures in different spatial frequencies. Three different type of measuring equipment have been used in this study. Non-contacting techniques are represented by Coherence Scanning Interferometry (CSI) and Confocal Microscopy (CM), while Atomic Force Microscopy (AFM) represents scanning probe microscopy techniques. For parameter evaluation the analysis software MountainsMap from DigitalSurf ${ }^{1}$ has been used.

The techniques used in this study are well described in literature $[16,17]$ and only a brief overview is given in this subsection. CSI is based on the idea to combine vertical scanning techniques with optical interferometry to achieve 3D surface measurement. For the measurements a CSI utilising a white light source were used - Vertical Scanning White Light Interferometer (MicroXam, ADE Phase shift Technologies ${ }^{2}$ ). The measurement area was approximately $165 \mu \mathrm{m} \times 125 \mu \mathrm{m}$ with a data sampling distance in $\mathrm{x}$ and $\mathrm{y}$ of $0,26 \mu \mathrm{m} \times 0,22 \mu \mathrm{m}$, and a vertical resolution of $0,9 \mathrm{~nm}$, which is assessed with consideration to experimentally measured noise level and topography repeatability of instrument. [18,19]

The confocal microscope has taken its name from the arrangement of the light path as the illumination and detection light paths share a common focal plane. This is achieved by two pinholes placed at the same distance from the specimen. Light goes throw a pinhole in a beamsplitter to reach the objective and the specimen. The beamsplitter works as a filter which reflects the excitation wavelength but is transparent for all others wavelengths. Therefore the emitted light from the surface can pass through the beamsplitter to the detector. As a consequence of the pinhole arrangement, the detected light mostly comes from the narrow focal plane which improves the vertical resolution of confocal microscopes. In this study the confocal microscope $\mu$ surf from Nanofocus ${ }^{3}$ has been used. The measurement area was $160 \mu \mathrm{m} \times 160 \mu \mathrm{m}$ with a data sampling distance in $\mathrm{x}$ and $\mathrm{y}$ of $0,15 \mu \mathrm{m} \times$ $0,15 \mu \mathrm{m}$, and a vertical resolution of $1 \mathrm{~nm}$. The absolute minimum resolutions for confocal instruments are currently being debated and e.g. R.Leach at al. in the book "Optical measurement of surface topography" on pp 274- 277 manufacturer states for 0,12 to $0,15 \mu \mathrm{m}$ for lateral resolution and limits of the noise level for confocal instrument in order of 0,3nm.

The AFM is a well-established technique with a higher lateral resolution compared to optical techniques, but the measurement acquisition is generally more time consuming. The AFM consists of a tiny tip made of silicon nitride or silicon which is mounted on a cantilever and moved by piezoelectric controller onto the surface to be measured. A laser beam reflected from the back of the cantilever and directed onto photodiode detector measures the vertical position of the tip as the surface is scanned. In this study a DS $95-504^{4}$ AFM was used. For the measurements Si-tip with curvature

\footnotetext{
${ }^{1}$ www.mountainsmap.com

2 http://cma.tcd.ie/misc/MicroXam.pdf

${ }^{3}$ http://www.nanofocus.com

${ }^{4}$ http://www.dme-spm.com
} 
radius $8-10 \mathrm{~nm}$ has been used. The measurement area was approximately $40 \mu \mathrm{m} \times 40 \mu \mathrm{m}$ and sampling points $1024 \times 1024$ with data sampling distance in $\mathrm{x}$ and $\mathrm{y}$ of $39 \times 39 \mathrm{~nm}$, and a vertical resolution of $0,3 \mathrm{~nm}$.

\subsection{Calculation of Power Spectral Density}

Some years ago, the optics and the microelectronics communities encountered the need to deal more incisively with topography data. It was found fruitful to Fourier transform the topography data to obtain the contribution at different lateral scales: the power spectral density (PSD). In this subsection the mathematical model used for calculation of two-dimensional PSD from surface height data is

presented. The PSD of a surface height map $h(x, y)$ is defined as [14, 20, 21]:

$$
\operatorname{PSD}\left(f_{x}, f_{y}\right)=\lim _{L \rightarrow \infty} \frac{1}{L^{2}}\left|\int_{-L / 2}^{L / 2} \int_{-L / 2}^{L / 2} h(x, y) e^{-2 \pi \mathrm{i}\left(f_{\mathrm{x}} x+f_{\mathrm{y}} y\right)} \mathrm{d} x \mathrm{~d} y\right|^{2},
$$

where $h(x, y)$ is the surface topography data, and $f_{\mathrm{x}}$ and $f_{\mathrm{y}}$ are rectangular components of the surface frequencies. Eq. (1) describes the relative contributions of all the possible surface spatial frequencies for an ideal measurement of an infinite surface in the limiting case from zero frequency (an infinite surface) to an infinite frequency (infinitely small structure) [8]. In practice, topographic images of surfaces are recorded in the form of digitized data of surface heights, which is finite rather than infinite and sampled rather than continuous. All samples used in this study were isotropic, and the PSD $\left(f_{\mathrm{x}}, f_{\mathrm{y}}\right)$ function has polar symmetry, so the calculation of PSDs has been performed in polar coordinates:

$$
\begin{gathered}
f=\left(f_{\mathrm{x}}^{2}+f_{\mathrm{y}}^{2}\right)^{1 / 2} \\
\theta=\tan ^{-1}\left(\frac{f_{\mathrm{x}}}{f_{\mathrm{y}}}\right),
\end{gathered}
$$

Averaging the resulting PSD $(f, \theta)$ over the azimuthal angle yield the two-dimensional isotropic PSD:

$$
P S D_{2 \mathrm{D}}(f)=\frac{1}{2 \pi} \int_{0}^{2 \pi} P S D(f, \theta) \mathrm{d} \theta .
$$

The rms roughness of each surface was calculated from the PSD using Eq. (4):

$$
\sigma^{2}=\int_{f \min }^{f \max } P S D_{2 \mathrm{D}}(f) f \mathrm{~d} f
$$

where $\left(f_{\min }, f_{\max }\right)$ is the spatial frequency interval in which the PSD is defined. All calculations have been performed using Matlab ${ }^{5}$ software. The PSD graphs are presented in log-log scale which is the most natural way to display the PSD-functions.

\section{Result and discussions}

\subsection{Comparison between PSDs obtained using different techniques}

This section presents a comparison between the results obtained by using different measurement techniques for characterising each sample surface. For practical reasons it was not possible to perform each measurement on the same exact position on each sample which means that the results will be influenced by non-uniformities over the surfaces. Still, the PSDs can give guidelines for the choice of suitable measuring techniques for the various surfaces.

Figure 1, 2 and 3 presents the PSDs for all six samples calculated for each individual measurement technique used. It can be seen that all instruments used in this study share a common surface spatial frequency interval of $0,2-4 \mu^{-1}$ as indicated with the dotted lines in the Figures1,2,3.This interval has been chosen to remove the influence and uncertainties concerning resolutions. Thus, RMS

\footnotetext{
${ }^{5}$ se.mathworks.com/products/matlab
} 
roughness values in this frequency interval is calculated and listed in Table 2, including the approximate standard deviation for each specific surface and instrument.

All topographic images of the surfaces were in the form of data of surface heights. The data from CSI and CM measurements used for PSD calculations were aligned by subtracting a fitted least square plane from the raw heights for each data set. The presence of a curvature was observed in the AFM measurements, which can be explained by the action of the piezoelectric scanner that moves the probe [3]. For this reason, measurements made by the AFM have been corrected by subtraction of a fitted line-by-line polynomial of $3^{\text {rd }}$ degree order although it can be noted that this only have a minor influence on the result compared to using a plane for alignment. The PSDs presented in Figures 1, 2 and 3 are mean PSDs based on three measurement on each sample and with each instrument.

Table 2. RMS roughness and corresponding standard deviations (in nm) calculated from PSD-plots in the spatial frequency interval $0,2-4 \mu \mathrm{m}^{-1}$.

\begin{tabular}{ccccccc}
\hline $\begin{array}{c}\text { Sample } \\
\text { Instruments }\end{array}$ & $\mathrm{R}_{3} \mathrm{n}_{2} \mathrm{G}_{4}$ & $\mathrm{R}_{3} \mathrm{n}_{1} \mathrm{G}_{4}$ & $\mathrm{R}_{2} \mathrm{n}_{2} \mathrm{G}_{3}$ & $\mathrm{R}_{1} \mathrm{n}_{2} \mathrm{G}_{3}$ & $\mathrm{R}_{1} \mathrm{n}_{2} \mathrm{G}_{1}$ & $\mathrm{R}_{1} \mathrm{n}_{1} \mathrm{G}_{1}$ \\
\hline AFM & $3 \pm 0,5$ & $2 \pm 0,5$ & $4 \pm 0,5$ & $15 \pm 5$ & $118 \pm 20$ & $99 \pm 20$ \\
CM & $4 \pm 0,5$ & $1 \pm 0,5$ & $20 \pm 5$ & $23 \pm 5$ & $132 \pm 10$ & $97 \pm 10$ \\
CSI & $4 \pm 0,5$ & $2 \pm 0,5$ & $38 \pm 10$ & $62 \pm 10$ & $207 \pm 10$ & $156 \pm 10$ \\
\hline
\end{tabular}

It can be seen that the RMS values for the glossiest surfaces $\left(G_{4}\right)$ are significantly lower than for the other surfaces, and also that all instruments give quite similar results for these two samples. As AFM has the highest lateral and vertical resolution, this technique can best describe the nano-features of these surfaces and therefore any further analysis has been based on the AFM measurements. For the samples $R_{2} n_{2} G_{3}$ and $R_{1} n_{2} G_{3}$ significant differences in RMS roughness can be observed between AFM, $\mathrm{CM}$ and CSI. Figure 2 shows that these samples have quite interesting topography with quite large lateral structures, and as the evaluation area for AFM measurements is about $40 \times 40 \mu \mathrm{m}$, these surfaces cannot be accurately captured by AFM without stitching several measurements together. The same reasoning, although less evident, also applies for samples $R_{1} n_{2} G_{1}$ and $R_{1} n_{1} G_{1}$. Therefore optical techniques are judged as being more suitable for analysing the samples with gloss levels $G_{1}$ and $G_{3}$.
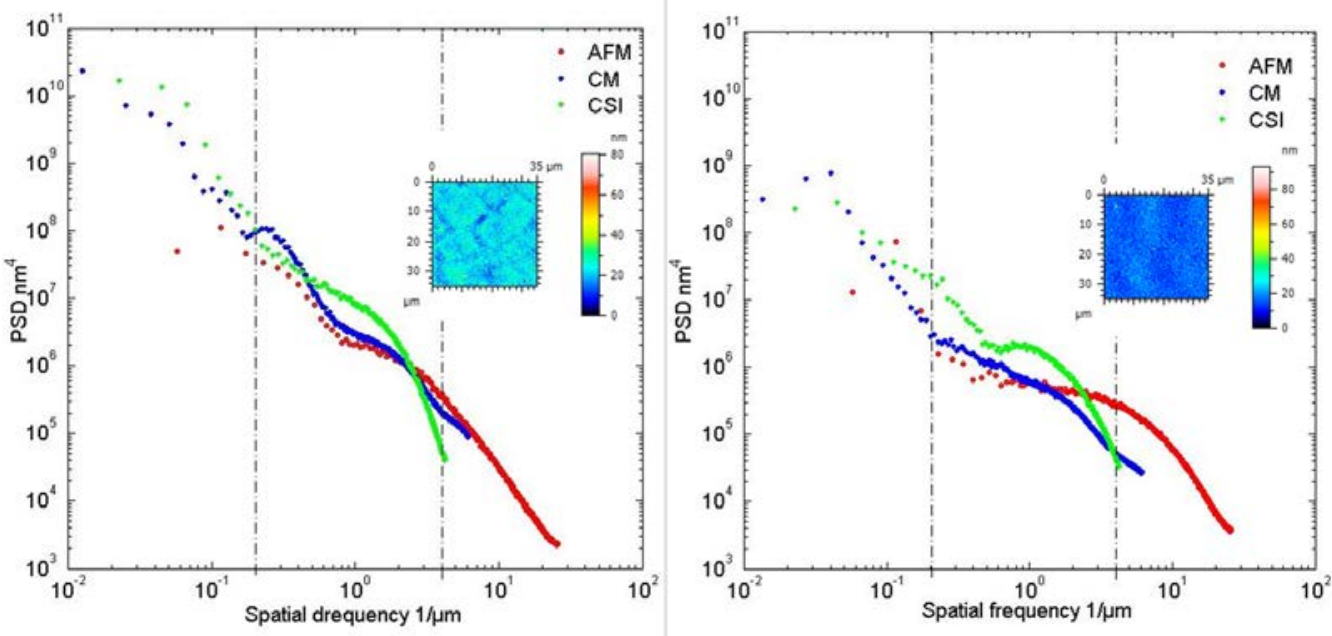

Figure 1. PSDs for the $\mathrm{R}_{3} \mathrm{n}_{2} \mathrm{G}_{4}$ sample (left) and the $\mathrm{R}_{3} \mathrm{n}_{1} \mathrm{G}_{4}$ sample (right). 

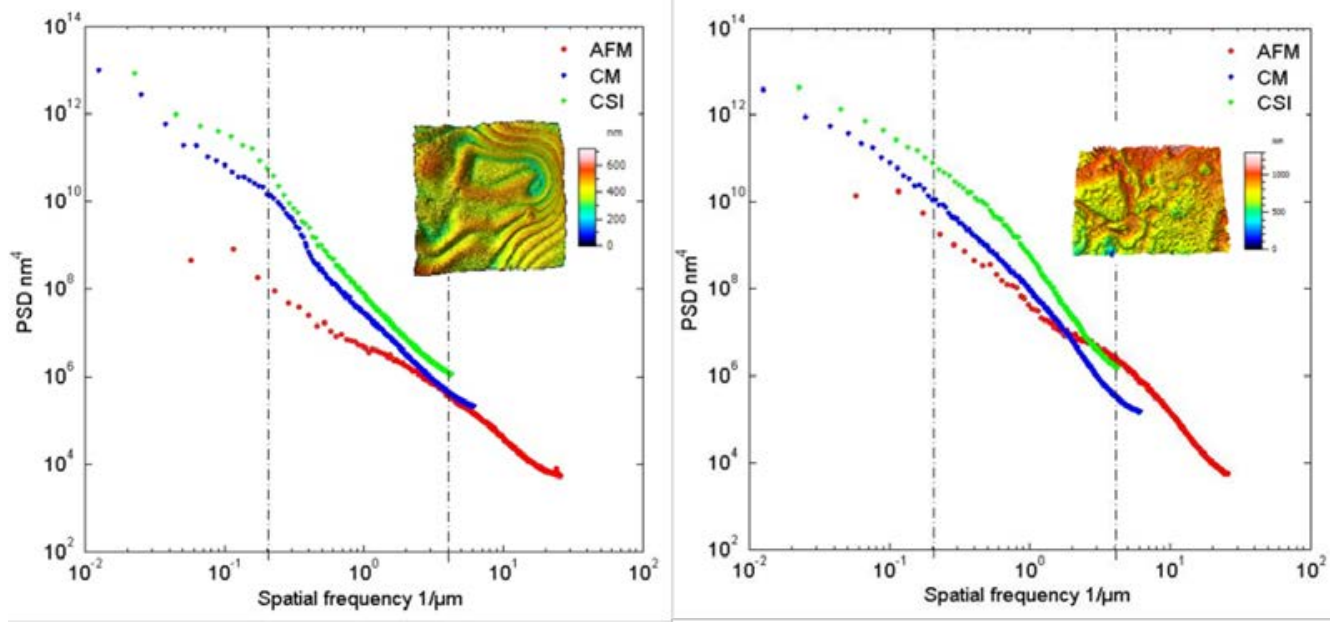

Figure 2. PSDs for the $\mathrm{R}_{2} \mathrm{n}_{2} \mathrm{G}_{3}$ sample (left) and $\mathrm{R}_{1} \mathrm{n}_{2} \mathrm{G}_{3}$ sample (right).
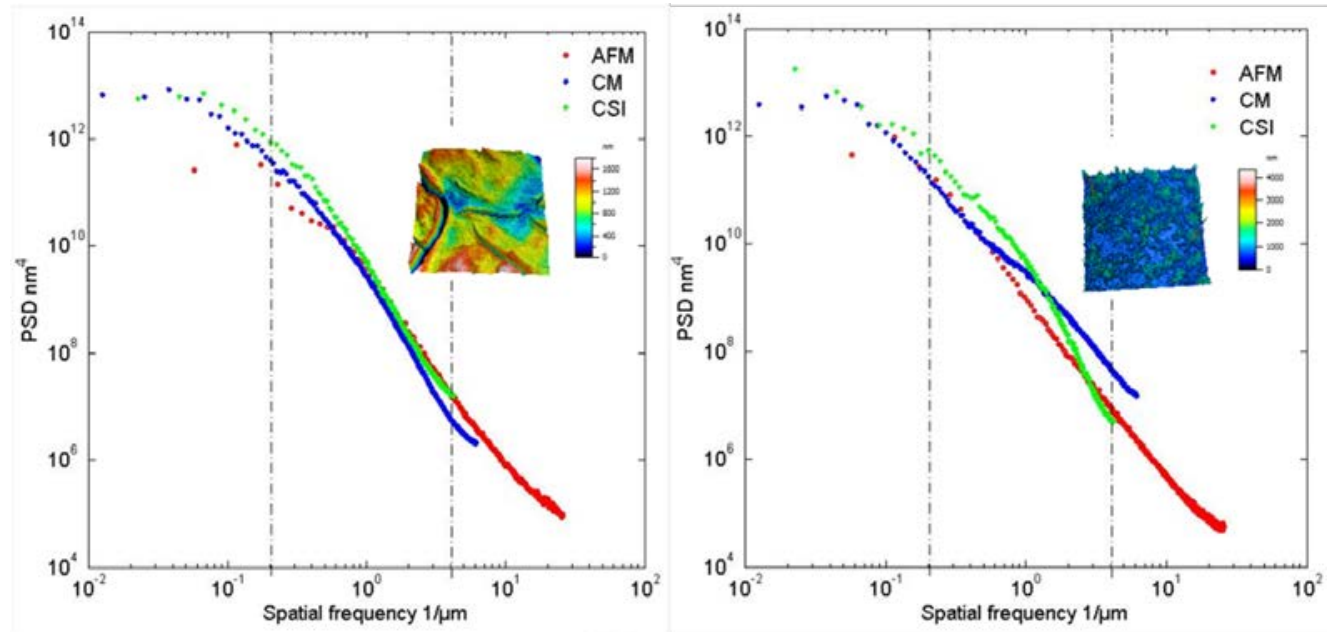

Figure 3. PSDs for the $\mathrm{R}_{1} \mathrm{n}_{2} \mathrm{G}_{1}$ sample (left) and the $\mathrm{R}_{1} \mathrm{n}_{1} \mathrm{G}_{1}$ sample (right).

Some consideration about sample $\mathrm{R}_{1} \mathrm{n}_{2} \mathrm{G}_{1}$ should be done. No obvious defects were found when visually inspecting the sample surface but when measurements were carried out and analysed, distinct cracks could be seen (Figure 3 - left). These unwanted features are believed to be an artefact from the manufacturing process and will not be present on the final samples to be used for the gloss scale. However, as no other similar sample was available at the time of this study the analysis was carried out using the existing data.

\subsection{PSD (Power spectral density) and Motif analysis of samples}

The samples examined in this study are designed not only with consideration of the overall gloss level but also considering different surface structures and amplitudes. The surface profiles shown in Figure 4 are intended to give an overview and depict these differences. Corresponding PSDs for each sample is shown in Figure 5 and 6. From these figures it is evident that the samples all differ quite significant in terms of structure and amplitude, although both surface non-uniformity as well as the measurement techniques used are affecting the results. However, reviewing the measured surface roughness over all available spatial frequencies, the samples can roughly be grouped into three different groups corresponding to the manufactured gloss levels $\left(G_{1}, G_{3}\right.$ and $\left.G_{4}\right)$ clearly correlated to the roughness type $\mathrm{R}_{1}, \mathrm{R}_{2}$ and $\mathrm{R}_{3}$. 
In the following section and in Table 3 , a pairwise comparison between the $G_{1}, G_{3}$ and $G_{4}$ samples are done. For example, reviewing the PSDs for the $G_{4}$ samples show a quite significant difference for lower frequencies where $R_{3} n_{1} G_{4}$ is clearly smoother than $R_{3} n_{2} G_{4}$. However, from the $A F M$ measurements it can be seen that for frequencies higher than about $5 \mu \mathrm{m}^{-1}$ the PSDs crosses meaning that $R_{3} n_{2} G_{4}$ is smoother on a very short wavelength scale. Although $R_{3} n_{2} G_{4}$ is slightly smoother in terms of $S_{q}$ in this limited frequency interval, it has higher peaks than $R_{3} n_{1} G_{4}$. It also has denser peaks but with a larger peak curvature on average. Whether these surface properties will have any significant impact on the perceived gloss remain to be seen, but the analysis is an example of using PSDs to identify possibly interesting spatial frequency intervals and apply corresponding filters to surface parameters. Similar analysis for the other two sample pairs $\left(\mathrm{G}_{1}\right.$ and $\left.\mathrm{G}_{3}\right)$ is also shown in Table 3.

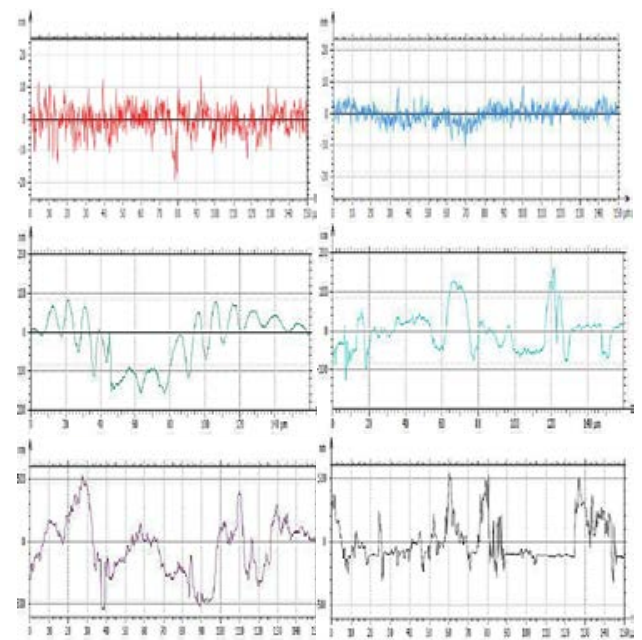

Figure 4. Surface profiles of the samples. Amplitude range: $50 \mathrm{~nm}$ for blue $\left(\mathrm{R}_{3} \mathrm{n}_{1} \mathrm{G}_{4}\right)$ and red $\left(\mathrm{R}_{3} \mathrm{n}_{2} \mathrm{G}_{4}\right) ; 400 \mathrm{~nm}$ for green $\left(\mathrm{R}_{2} \mathrm{n}_{2} \mathrm{G}_{3}\right)$ and cyan $\left(\mathrm{R}_{1} \mathrm{n}_{2} \mathrm{G}_{3}\right)$; $1200 \mathrm{~nm}$ for magenta $\left(\mathrm{R}_{1} \mathrm{n}_{2} \mathrm{G}_{1}\right)$ and black $\left(\mathrm{R}_{1} \mathrm{n}_{1} \mathrm{G}_{1}\right)$.Profile length $150 \mu \mathrm{m}$.

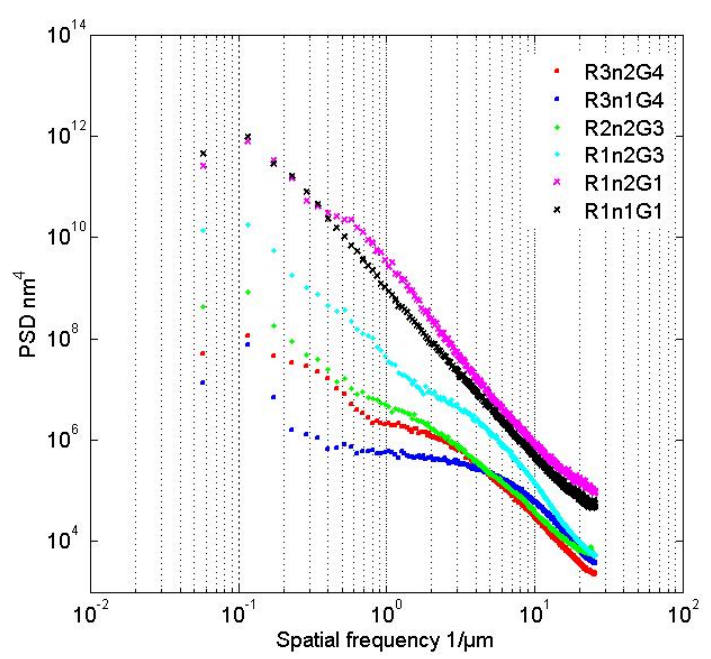

Figure 5. PSDs calculated from AFM measurements.

Table 3. Surfaces parameters with corresponding standard deviation calculated from measurements using different instruments.

\begin{tabular}{lcccccc}
\hline Instrument & \multicolumn{2}{c}{ AFM } & \multicolumn{2}{c}{ CSI } & \multicolumn{2}{c}{ CM } \\
\hline Sample & $\mathrm{R}_{3} \mathrm{n}_{2} \mathrm{G}_{4}$ & $\mathrm{R}_{3} \mathrm{n}_{1} \mathrm{G}_{4}$ & $\mathrm{R}_{1} \mathrm{n}_{1} \mathrm{G}_{1}$ & $\mathrm{R}_{1} \mathrm{n}_{2} \mathrm{G}_{1}$ & $\mathrm{R}_{2} \mathrm{n}_{2} \mathrm{G}_{3}$ & $\mathrm{R}_{1} \mathrm{n}_{2} \mathrm{G}_{3}$ \\
\hline Height & & & & & & \\
Parameters & & & & & & \\
$\mathrm{S}_{\mathrm{q}}(\mathrm{nm})$ & $5 \pm 0,2$ & $4 \pm 0,4$ & $100 \pm 8$ & $102 \pm 6$ & $277 \pm 25$ & $201 \pm 42$ \\
$\mathrm{~S}_{\mathrm{z}}(\mathrm{nm})$ & $96 \pm 16$ & $31 \pm 1$ & $466 \pm 76$ & $1070 \pm 186$ & $1663 \pm 174$ & $1346 \pm 334$
\end{tabular}



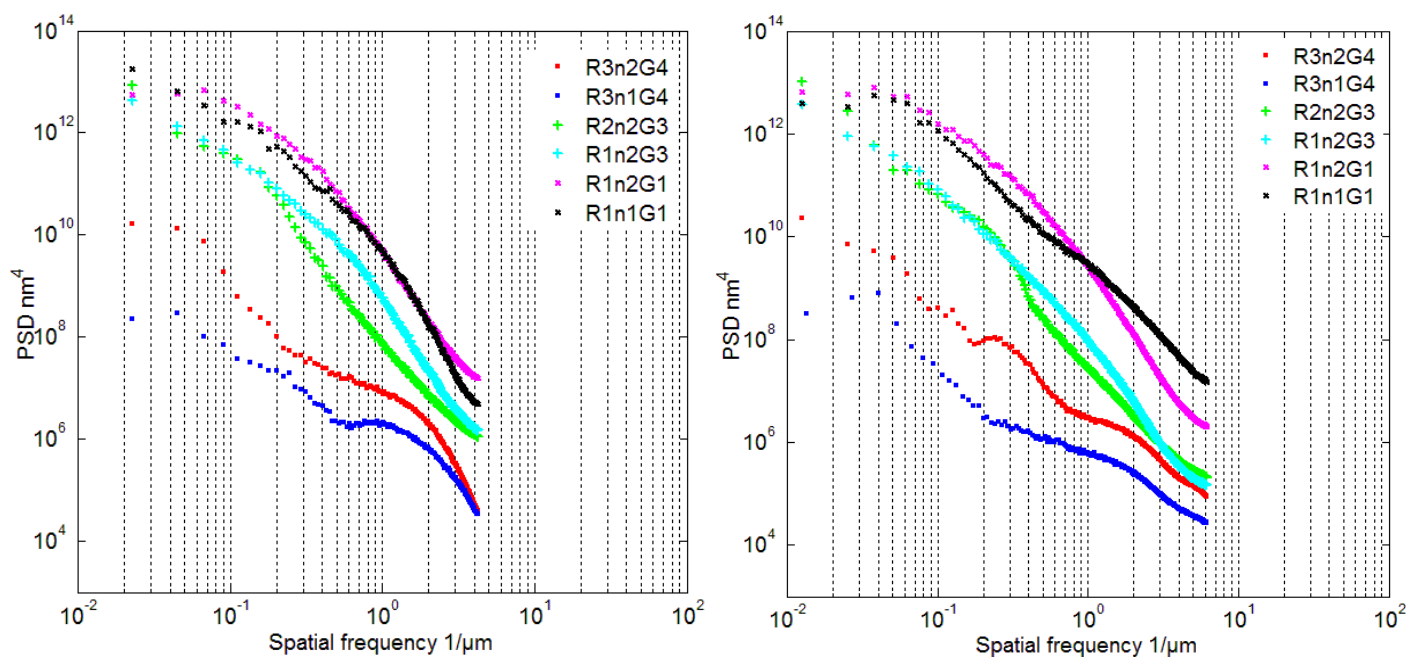

Figure 6. PSDs for all samples, measurements by CSI (left) and CM (right).

Feature parameters like density of peaks, area of peaks and peak curvature are important as they have been found to play a significant role for many functional surfaces regarding wear and abrasion, but could possibly also be of importance for a surface's appearance. Normally significant features are identified by segmentation of a surface and selected by a discrimination method (pruning). This separation of various surface features can either be calculated using the ISO 25178-2:2012 parameters $\mathrm{S}_{\mathrm{pd}}$ and $\mathrm{S}_{\mathrm{pc}}$ or alternatively using the areal motif analysis [22,23]. Motif analysis is performed with consideration to the maximum peak height $\left(\mathrm{S}_{\mathrm{z}}\right.$ parameter $)$, which determines the minimum height of the motif. This method gives a visual and robust feedback for the detection of motifs in the analysis and has been chosen in this study to calculate number of peaks, peak area and peak height, see figure 7 -9 below.
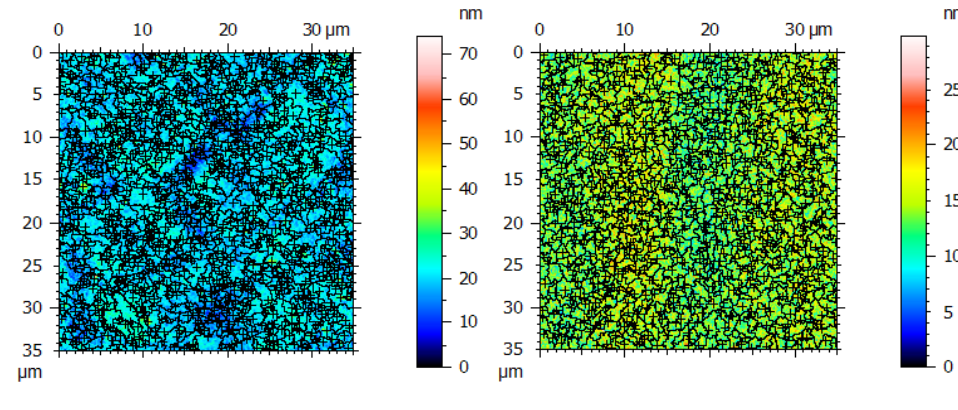

\begin{tabular}{|c|c|c|c|c|c|c|c|}
\hline Number of motifs & 981 & & & Number of motifs & 649 & & \\
\hline Parameters & Stat. & Value & Unit & Parameters & Stat. & Value & Unit \\
\hline Height & Mean & 12.47 & $\mathrm{~nm}$ & Height & Mean & 10.2 & $\mathrm{~nm}$ \\
\hline Area & Mean & 1.252 & $\mu \mathrm{m}^{2}$ & Area & Mean & 1.89 & $\mu m^{2}$ \\
\hline
\end{tabular}

Figure 7. Motif analysis of AFM measured samples $\mathrm{R}_{3} \mathrm{n}_{2} \mathrm{G}_{4}$ (left) and $\mathrm{R}_{3} \mathrm{n}_{1} \mathrm{G}_{4}$ (right). 


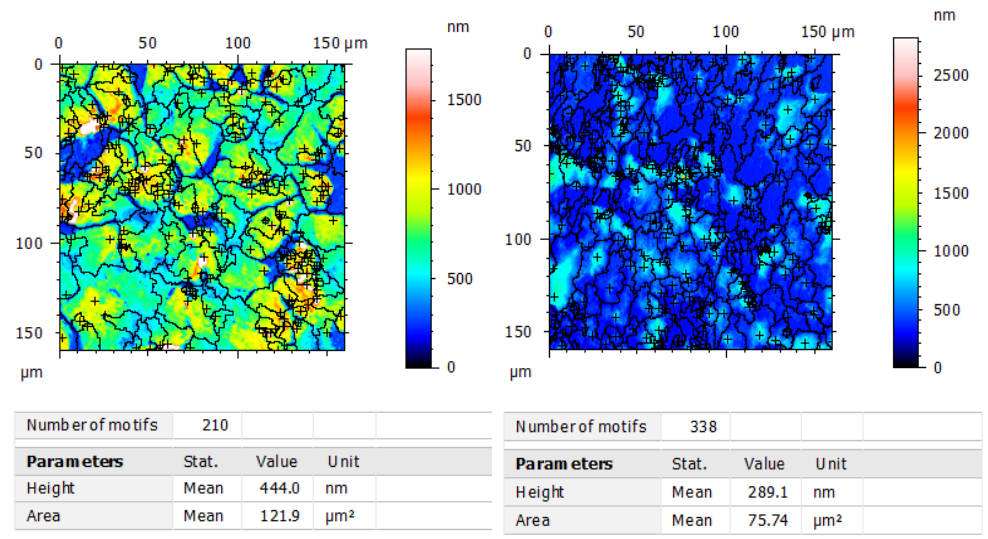

Figure 8. Motif analysis of $C M$ measured samples $R_{1} n_{1} G_{1}$ (left) and $\mathrm{R}_{1} \mathrm{n}_{2} \mathrm{G}_{1}$ (right).
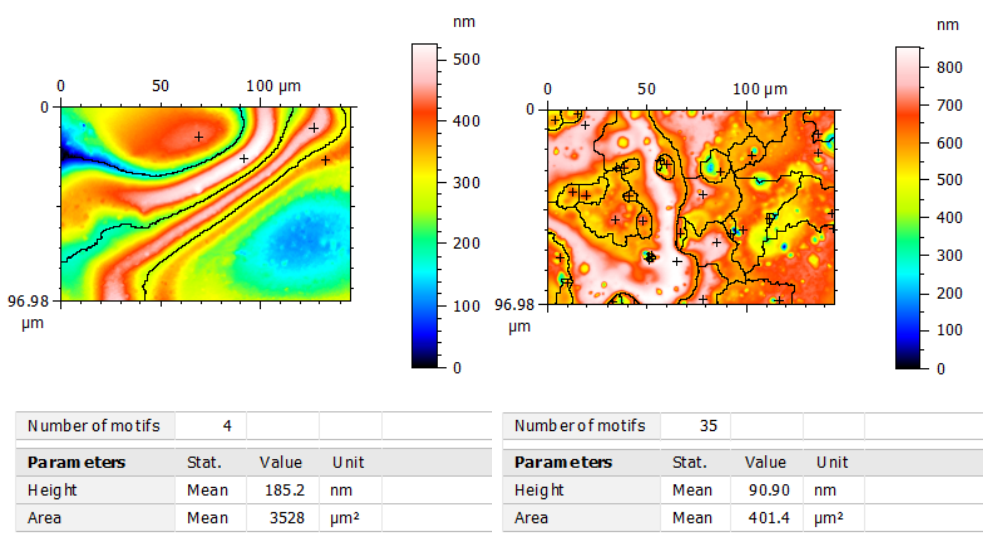

Figure 9. Motif analysis of CSI measured samples $\mathrm{R}_{2} \mathrm{n}_{2} \mathrm{G}_{3}$ (left) and $\mathrm{R}_{1} \mathrm{n}_{2} \mathrm{G}_{3}$ (right).

The criterion for the segmentation for the motif analysis for all the samples was to decide the smallest motif height as $10 \%$ of Sz within measurements within the same gloss "class" $\left(\mathrm{G}_{1^{-}}, \mathrm{G}_{3^{-}}\right.$, and $\mathrm{G}_{4}$ surfaces). 


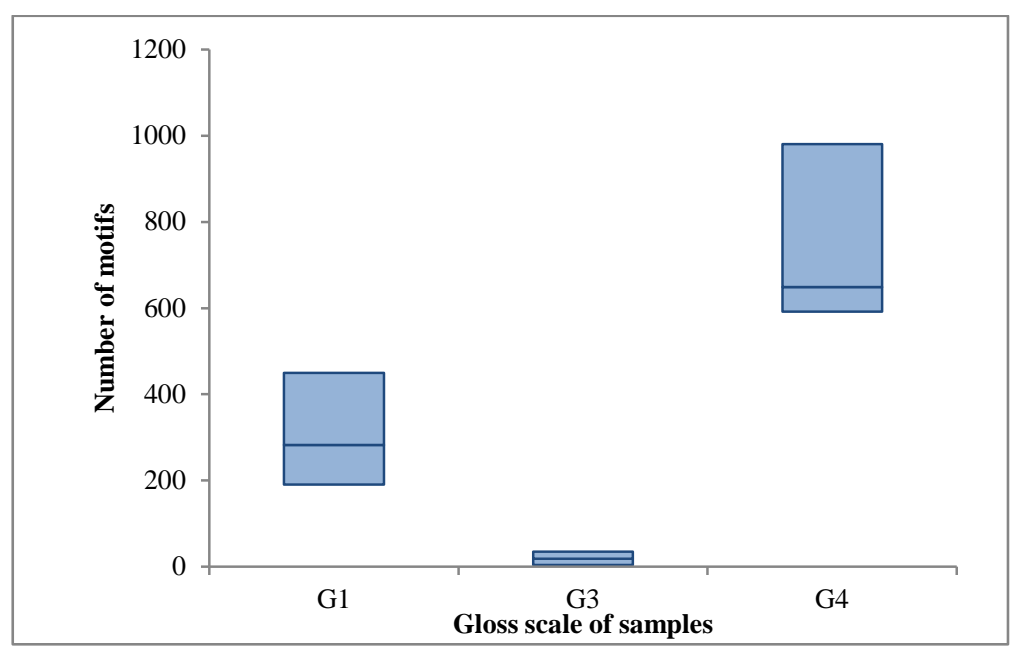

Figure 10. Number of motifs and gloss scale for the measurements performed by different techniques. Boxes in the plot are limited by max and min value of motifs and line in the box represent median value of motif for each gloss type.

Figure 10 shows nonlinear correlation between number of motifs and gloss scale of samples. No correlation between all the gloss scales is evident. However, a clear trend between number of motifs and gloss between $G_{1}$ and $G_{4}$ samples is shown but the $G_{3}$ samples characteristic long wavelength structure does not fit into the same "gloss model" relating the motifs to the gloss.

\section{Conclusions}

Six samples with different refractive indices, different gloss levels and different roughness types have been examined. In order to capture important surface structures that may contribute to the perceived surface glossiness, three different instrument utilising different measurement techniques were used, to investigate the suitability of each technique for the different surface types.

- The usage of PSD analysis is a strong tool for identification of the differences and equalities between instruments measuring similar topographies enabling a selection of the proper instruments for measuring at given frequencies or combination of frequencies.

- The analysis based on PSD and motif analysis reveals the surface topography frequency content as well as the number, height and area of significant features.

The selected samples were shown to have very specific and different surface characteristics originating from their manufacturing processes.

- The overall gloss levels of the samples $\left(G_{1}, G_{3}\right.$ and $\left.G_{4}\right)$ were in agreement with the manufacturing tolerances although differences where identified between sample pairs with the same nominal gloss level depending on the variation in spatial frequency content.

- The roughness's of the studied samples (R1, R2 and R3) were shown to closely correlate to the gloss levels due to specifics in the manufacturing process.

- Varying refractive indices on samples did not show any correlation to surface roughness.

The PSD analysis was complimented by motif analysis in different spatial frequency intervals. In order to give additional information regarding possibly important nano- and micro-features of the samples. Parameters such as peak area, and total number of peaks were examined.

- No clear linear correlation was established between the feature parameters and the gloss of the samples.

Further studies will continue the investigation of influence of surface structure on gloss of samples. 


\section{Acknowledgements}

The authors wish to thank St-Gobain Company - for their contribution in designing and manufacturing the samples, and NanoFocus for help with the measurements using Confocal Microscope. The authors are also thankful for the collaboration with Digital Surf through the MountainMap program. This work has been partially funded by the European Metrological Research Program (EMRP), The EMRP is jointly funded by the EMRP participating countries with EURAMET and the European Union.

\section{References}

[1] W Ji, MR Pointer, RM Luo, J Dakin. (2006). Gloss as an aspect of the measurement of appearance. Journal of the Optical Society of America, 23, 22-33.

[2] MS Landy. (2007). A gloss on surface properties. Nature, 447, 158-159.

[3] DJ Whitehouse, DK Bowen, VC Venkatesh, P Lonardo, CA Brown. (1994). Gloss and Surface Topography. Annals of the CIRP, Vol. 43, 541

[4] G Ged, G Obein, Z Silvestri, GL Rohellec, F Viénot. (2010) Recognizing real materials from their glossy appearance. Journal of Vision 10(9):18, 1-17.

[5] F Leloup, G Obein, M Pointer. (2014). Colour Research \& Application, 39, 6, 559-570, doi: 10.1002/col.21846

[6] A.C.Chadwick, R.W. Kentridge. (2015). The perception of gloss: A review. Vision Research 109, 221-235.

[7] Jean Bennett and Lars Mattsson, (1999) Introduction to Surface Roughness and Scattering. (Washington, DC :Optical Society of America).

[8] L.Qi, M.J.Chantler, J.P.Siebert, J.Dong, (2012). How mesoscale and microscale roughness affect perceived gloss. In Predicting perceptions: Proceedings of the \#rd international conference on appearance. pp 48-51. Edingburg, Scotland:Lulu Press,Inc.

[9] Y.-X.Ho, M.S.landy, L.T.Maloney. (2008). Conjoint measurement of gloss and surface texture. Psycological Science, 19(2), 196-204.

[10] M.Dietzsch, K.Papenfuß, T.Hartmann. (1998). The MOTIF-method (ISO 12085) - a suitable description for functional, manufactural and metrological requirements. Int. J. Mach.Tools Manufac. Vol 38, pp625-632.

[11] ISO 12085, Geometrical Product Specification (GPS) - Surface Texture: Profile Method Motif Parameters, 1996.

[12] S.Lou, X. Jiang, P.J. Scott. (2013). Correlating motif analysis and morphological filters for surface texture analysis. Measurement, 46, 993-1001.

[13] http://www.xdreflect.eu

[14] A Duparré, J Ferre-Borrull, S Gliech, G Notni, J Steinert, JM Bennett. (2002). Surface characterization techniques for determining the root-mean-square roughness and power spectral densities of optical components. Applied optics, Jan 1;41(1):154-71.

[15] G Ged, E Garre, J Teisseire, ME Himbert, G Obein, (2014) Development of a controlled metrological gloss scale. Conference: Newrad 2014, At Espoo, Finland

[16] R.Leach (2011) Optical measurement of surface topography. (Berlin: Springer) pp.71-106, 187208.

[17] DJ Whitehouse (1994) Handbook of surface metrology. (London: Institute of Physics Publising) pp.453-531.

[18] CL Ciusca, R Leach (2013) Measurement good prictice guide No 127 Calibration of the methrological characteristics of coherence scanning interferometers (CSI) and phase shift interferometers (PSI). ISSN 1368-6550 pp.17-19

[19] ISO 12085, Geometrical Product Specification (GPS) - Surface Texture: Areal-Part 604: Nominal characteristics of non-contact (coherence scanning interferometry) instruments, 2013.

[20] E Sidick (2009) Power spectral density specification and analysis of large optical surfaces. Proc. 
SPIE 7390, Modeling Aspects in Optical Metrology II, 73900L; http://dx.doi.org/10.1117/12.823844, doi:10.1117/12.823844.

[21] Jean Bennett and Lars Mattsson, (1999) Introduction to Surface Roughness and Scattering. (Washington, DC :Optical Society of America).

[22] ISO 25178-2:2012 Geometrical Product Specification (GPS)- Surface texture: Areal-Part 2:Terms, definitions and surface texture parameters

[23] R.Leach (2013) Characterisation of areal surface texture (Berlin: Springer) pp.45-65. 